\title{
Microvascular functions in patients with fibromyalgia syndrome: effects of physical exercise
}

\author{
Emre Esen, ${ }^{1}$ Alp Çetin ${ }^{2}$ \\ ${ }^{1}$ Department of Physical Medicine and Rehabilitation, Bilecik State Hospital, Bilecik, Turkey \\ ${ }^{2}$ Department of Physical Medicine and Rehabilitation, Medical Faculty of Hacettepe University, Ankara, Turkey \\ Received: March 2016 Accepted: September 2016
}

\begin{abstract}
Objectives: This study aims to evaluate the current state of microvascular function and to investigate the effect of supervised aerobic exercise on microvascular control mechanisms and health outcomes in women with fibromyalgia syndrome (FMS).

Patients and methods: Forty female patients (mean age $51 \pm 11$ years) with a diagnosis of FMS according to the American College of Rheumatology criteria and 20 healthy female controls (mean age $52 \pm 9$ years) were included in the study. Microvascular blood flow was measured using a laser Doppler flowmeter (LDF) at the volar skin site of the forearm. Pain severity and FMS were assessed using the visual analog scale (VAS) and Fibromyalgia Impact Questionnaire (FIQ), respectively, both at the beginnig and at the end of the study. Fibromyalgia was evaluated and a spectral analysis of LDF signals was carried out to assess the relative contribution of each control mechanisms. The local thermal hyperemia was used to test the microvascular functions. Moderate-intensity aerobic activity (energy expenditure 3.0 to 6.0 metabolic equivalent) was performed by treadmill walking for $30 \mathrm{~min}$ for five days each week for a month.

Results: The patients with FMS had lower VAS and FIQ scores at the end of the exercise period. There was a positive correlation between improved myogenic and neurogenic mechanisms and reduced FIQ scores. Cardiac signals were positively correlated with the FIQ scores at the end of the exercise period. Endothelial function was under the influence of pain, and baseline nitric oxide activity was found to be positively correlated with VAS.

Conclusion: Our study results suggest that microvascular functions are impaired in FMS patients and moderate exercise training is effective to improve the FIQ/VAS scores and enhance vascular functions.
\end{abstract}

Keywords: Cutaneous circulation; fibromyalgia; physical exercise; spectral analysis.

Fibromyalgia syndrome (FMS) is a widespread disabling pain disorder ${ }^{[1]}$ Patients with FMS who are unable to remain active due to pain exacerbations become increasingly deconditioned, and their ability to tolerate exercise is impaired over time. ${ }^{[2,3]}$ Due to the depressed mood with chronic pain, most women with FMS are physically inactive compared to age-matched healthy women. ${ }^{[4]}$ Lifestyle changes in FMS patients are of special interest, as physical inactivity has been proposed as an independent cardiovascular risk factor. ${ }^{[5]}$ It has been also shown that inactivity increases vascular nicotinamide adenine dinucleotide phosphate (NADPH) oxidase expression and activity ${ }^{[6]}$ and enhances vascular reactive oxygen species (ROS) production, which contributes to endothelial dysfunction during sedentary lifestyle, as opposed to a physically active lifestyle. ${ }^{[6,7]}$ On the other hand, beginning an exercise program at any stage of life can yield significant cardiovascular health benefits. ${ }^{[8]}$ Moreover, aerobic physical exercise is a common recommendation in the management of FMS and is thought to be a more helpful treatment for FMS than many pharmacological treatments. ${ }^{[9,10]}$

Due to the presence of a strong correlation between pain and skin hyperthermia, and the evidences showing that nitric oxide (NO) enhances the sensitivity of peripheral nociceptors, it has been suggested that pain

Corresponding author: Emre Esen, MD. Bilecik Devlet Hastanesi Fiziksel Tıp ve Rehabilitasyon Kliniği, 11040 Bilecik, Turkey. e-mail: emreesen81@gmail.com 
is associated with an excessive local level of $\mathrm{NO}^{[11]}$ An increased nitric oxide synthase (NOS) enzyme activity ${ }^{[12]}$ and high NO levels ${ }^{[13]}$ were reported in FMS patients. Larson et al. ${ }^{[14]}$ showed that intensity of pain experienced in patients with FMS was associated with the increased synthesis of NO. Although NO released from the vascular endothelium plays an important role in the regulation of vascular tone, the effect of extravascular overproduction of NO on blood flow control in FMS patients is still unknown.

In recent years, the measurement of human cutaneous circulation with laser Doppler flowmetry (LDF) has emerged as an excellent method to examine the mechanisms of microcirculatory function and dysfunction. ${ }^{[15-17]}$ As a result, cutaneous circulation has been used as a model circulation to investigate microcirculatory function in a variety of disease states, including FMS. ${ }^{[15]}$ Abnormal microcirculation and reduced blood flow have been reported in the skin above tender points in FMS patients. ${ }^{[18]}$ Morf et al., ${ }^{[19]}$ using intravital capillaroscopy in FMS patients, found morphological abnormalities indicating capillary dilatation, indicating functional impairment in microcirculation in this patient population. However, it has been suggested that the disturbed microcirculation in FMS may be due to abnormal regulation of capillary blood flow rather than morphological changes in the capillaries. ${ }^{[20]}$ The results of the current literature examining the effects of nociceptive C-fiber stimulation on microcirculation are controversial. Al-Allaf et al. ${ }^{[21]}$ found no significant differences in cutaneous microvascular reactivity between patients with FMS and controls. However, in another study, painful stimulation of latent myofascial trigger points attenuated skin blood flow response in healthy individuals, suggesting an increased vasoconstrictor activity. ${ }^{[22]}$ Although frequency domain methods based on fast Fourier transform (FFT) may provide more valuable physiological insights into the individual control mechanisms of blood flow, ${ }^{[23]}$ aforementioned few studies on microcirculation in FMS patients used simple time domain indices. However, blood flow in the microvascular bed shows oscillations of different origins, which indicates the current functional state of the blood flow control systems. To date, six control systems have been described in the frequency ranging between 0.005 and $2 \mathrm{~Hz}:{ }^{[24]}$ endothelial related metabolic activity (0.005-0.0095 Hz); NO-dependent endothelial activity $(0.0095-0.021 \mathrm{~Hz})$ : sympathetic neurogenic activity $(0.021-0.052 \mathrm{~Hz})$; intrinsic myogenic activity (0.052-0.145 Hz); respiratory activity $(0.145-0.6 \mathrm{~Hz})$ and heartbeat $(0.6-2 \mathrm{~Hz})$. The current state of these control mechanisms has not been studied in FMS patients, yet.

In the present study, we aimed to evaluate the current state of microvascular function and to investigate the effect of supervised aerobic exercise on microvascular control mechanisms and health outcomes in women with FMS.

\section{PATIENTS AND METHODS}

This prospective, controlled study included 40 postmenopausal women with FMS (mean age $51 \pm 11$ years) and 20 healthy controls (mean age $52 \pm 9$ years) between March 2013 and 26 April 2013. Patients with a history of hypertension, diabetes, coronary/peripheral artery disease, congestive heart failure, smoking, and obesity (body mass index $>30 \mathrm{~kg} / \mathrm{m}^{2}$ ) were excluded from the study. Those with inflammatory rheumatic diseases, severe psychiatric illnesses, and respiratory or cardiovascular diseases which affected the blood flow were also excluded. The study protocol was approved by the institutional Ethics Committee. The study was conducted in accordance with the principles of the Declaration of Helsinki. A written informed consent was obtained from each participant.

All patients filled out the Turkish version ${ }^{[25]}$ of the Revised Fibromyalgia Impact Questionnaire (FIQR), ${ }^{[26]}$ which is a validated and self-administered tool. This tool was used to evaluate the patient based on the severity of three linked sets of domains: physical function, overall impact, and symptoms. The total maximum score is 100 . In addition, pain severity was assessed using the visual analog scale (VAS).

Initially, all patients and controls were screened for the recruitment. Exercise program was completed for all patients as of June 2013. Some patients did not regularly participate to the exercise program. Therefore, the data of these patients were excluded from the analysis. Although 40 patients participated, the number of patients who was included in the analysis was 20 . Baseline characteristics and reliability of questionnaire data are summarized in Table 1.

A data acquisition system (Biopac Systems Inc., California, USA) equipped with a laser Doppler flowmeter $(780 \mathrm{~nm}, 1 \mathrm{~mW})$ was used to record the forearm cutaneous blood flow. To record the blood perfusion in the center of a locally heated area of skin, the fibers of the LDF probe $(480 \mu \mathrm{m}$ diameter $)$ were placed in the center of a heating probe. The heating unit (Moor Instruments Ltd., Devon, UK) was able to control the temperature of the probe 
Table 1. Baseline characteristics of the patients and healthy controls

\begin{tabular}{|c|c|c|c|c|c|c|}
\hline \multirow[t]{2}{*}{ Characteristics } & \multicolumn{2}{|c|}{ FMS patients } & \multicolumn{2}{|c|}{ Healthy controls } & \multirow[t]{2}{*}{ Type of comparison test } & \multirow[b]{2}{*}{$p$} \\
\hline & $\mathrm{n}$ & Mean \pm SD & $\mathrm{n}$ & Mean \pm SD & & \\
\hline \multicolumn{7}{|l|}{ Sex } \\
\hline Males & - & & - & & & \\
\hline Females & 40 & & 20 & & & \\
\hline Age (years) & & $51 \pm 11$ & & $52 \pm 9$ & Wilcoxon matched pair & 0.7314 \\
\hline Weight $(\mathrm{kg})$ & & $71.9 \pm 11.3$ & & $70.5 \pm 7.2$ & Wilcoxon matched pair & 0.4796 \\
\hline Body mass index $\left(\mathrm{kg} / \mathrm{m}^{2}\right)$ & & $28.0 \pm 4.9$ & & $26.0 \pm 2.0$ & Wilcoxon matched pair & 0.6308 \\
\hline Diastolic blood pressure (mmHg) & & $83.7 \pm 7.9$ & & $77.9 \pm 7.4$ & Wilcoxon matched pair & 0.2471 \\
\hline Systolic blood pressure $(\mathrm{mmHg})$ & & $126.4 \pm 19.8$ & & $124.4 \pm 14.9$ & Wilcoxon matched pair & 0.4925 \\
\hline Heart rate (beats/min) & & $68.9 \pm 7.3$ & & $69.6 \pm 7.4$ & Wilcoxon matched pair & 0.8101 \\
\hline FIQ Score & & $69.7 \pm 12.5$ & & - & $\begin{array}{c}\text { Reliability test } \\
\text { Cronbach's alpha }=\end{array}$ & 0.714 \\
\hline Pain (Visual Analog Scale) & & $8.4 \pm 1.5$ & & - & $\begin{array}{l}\text { Reliability test } \\
\text { Cronbach's alpha }=\end{array}$ & 0.736 \\
\hline
\end{tabular}

FIQ, Fibromyalgia Impact Questionnaire; SD: Standard deviation.

with $\pm 0.3^{\circ} \mathrm{C}$ accuracy. This combined probe was fixed to the forearm skin with a double-sided adhesive tape.

Cutaneous blood flow was recorded from the volar site of forearm of the subjects lying in supine position. The studies were performed in a quiet room at $23 \pm 2{ }^{\circ} \mathrm{C}$. All participants were asked to refrain from consuming alcohol and caffeine containing drinks a day before the measurements. Each had $30 \mathrm{~min}$ rest before the test. After a $15 \mathrm{~min}$ recording of the baseline skin blood flow, a constant local heat $\left(42^{\circ} \mathrm{C}\right)$ was applied ${ }^{[27]}$ to test the local control mechanisms of cutaneous vascular beds. The LDF signal was recorded during the peak vasodilator response to local heating at least for $15 \mathrm{~min}$. Cutaneous blood flow was recorded before the beginning of the exercise training protocol and at the end of the training program. Figure 1 illustrates the experimental design of the blood flow measurements.
Although physical inactivity increases oxidative stress and endothelial dysfunction, ${ }^{[6]}$ intense physical training decreases circulating antioxidants and endothelium-dependent vasodilatation; ${ }^{[28]}$ therefore, it is necessary to optimize exercise training in FMS patients. It has been suggested that increasing the frequency of exercise, rather than intensity, may be useful $^{[29]}$ and optimal intensity of training is attained with moderate-intensity exercise. ${ }^{[30]}$ In addition, it has been shown that a single bout of exercise improves endothelium-dependent dilation for about two days with a peak effect after 12-24 h and, regular exercise further improves adaptation and slowly returns to sedentary levels within a week of detraining. ${ }^{[31]}$ Therefore, moderate-intensity aerobic activity was performed in this study.

Moderate-intensity aerobic activity, which is equivalent to a brisk walk (energy expenditure

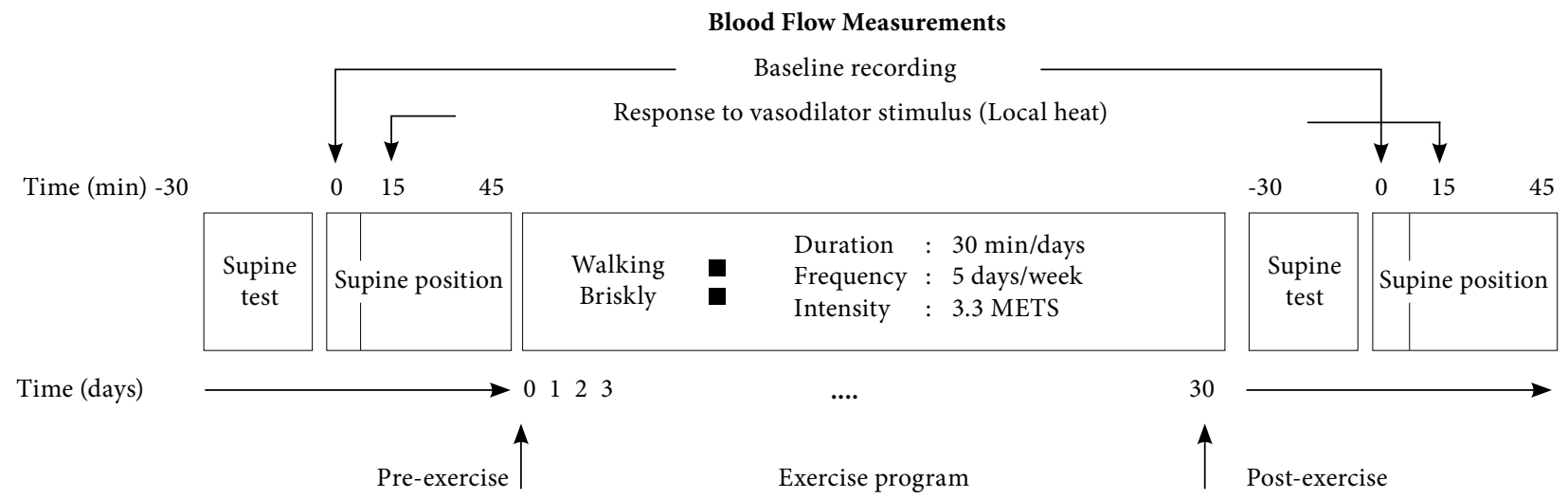

Figure 1. Experimental design for determining the effect of physical exercise program on microvascular blood flow control mechanisms. 
3.0 to 6.0 metabolic equivalent: METs ${ }^{[32]}$ and noticeably accelerates the heart rate (HR) $(70-85 \%$ of the age predicted maximum of $\mathrm{HR})^{[10]}$ was performed by treadmill walking for $30 \mathrm{~min}$ for five days each week during a month under supervision of a physiatrist (Figure 1). The patients were instructed to walk as quickly as possible, but not to run. Exercise begun at low-intensity, then gradually increased according to the individual tolerance of each patient. ${ }^{[33]}$ The walking distance, time, speed, energy expenditure in calorie, and HR were read from the panel of the treadmill.

Data relating to LDF was collected at 200 samples per sec. To find the frequency content of the LDF signals, spectral analysis was performed using the Acknowledge software (Biopac Systems Inc., California, USA) with its remove trend and windowing "Blackman" functions. This tool calculated the power spectral density (PSD) of LDF signals in [blood perfusion unit (BPU)]2/Hz, using the FFT algorithm. Then, the integral of the calculated PSD spectrum of the LDF signal was normalized to unity to standardize the spectrum. As the data length required in the FFT algorithm was equal to exact power of two, we used $2^{17}$ data points in our calculations. As a result, the frequency components from $200 / 2^{17}=$ $0.0015 \mathrm{~Hz}$ to $100 \mathrm{~Hz}$ were plotted in this analysis. However, we used six subintervals: ${ }^{[23]}$ endothelial related metabolic activity (VI: 0.005-0.0095 Hz); NO-dependent endothelial activity (V: 0.0095-0.021 $\mathrm{Hz}$ ): sympathetic neurogenic activity (IV: 0.021-0.052 $\mathrm{Hz}$ ); intrinsic myogenic activity (III: $0.052-0.145 \mathrm{~Hz}$ ); respiratory activity (II: $0.145-0.6 \mathrm{~Hz}$ ), and heart beat (I: $0.6-2 \mathrm{~Hz}$ ). In addition to the relative contribution of above each control mechanism to total PSD, we analyzed changes with vasodilatation before and after exercise.

\section{Statistical analysis}

The primary outcome measure for this study was the blood flow measured with LDF. Esen et al. ${ }^{[17]}$ reported that the standard deviation (SD) of the parameters calculated from the LDF signal was 0.1, and we also used this value to find the sample size of each group using the two-tailed unpaired t-test. Our sample size calculation indicated that 20 participants were needed to obtain SD of this magnitude using a power of 0.95 and alpha $(\alpha)$ level of 0.05 (StateMate software, GraphPad Software Inc., CA, USA).

Secondary outcome measure of this study was the FIQ score. Since the scatter of the FIQ score is unknown, we used SD of the differences of completed study to determine the power. Our study ( $\mathrm{n}=20$ and SD of differences $=14.16$ ) had a $95 \%$ power to detect a smallest mean difference between the pairs of 8.55 with a significance level $(\alpha)$ of $0.01 \mathrm{using}$ the two-tailed paired t-test.

Tertiary outcome measure of this study was the VAS score. Our study ( $\mathrm{n}=20$ and SD of differences $=2.7$ ) had a $95 \%$ power to detect a smallest mean difference between the pairs of 2.55 with a significance level $(\alpha)$ of 0.01 using the two-tailed paired t-test.

Internal consistency of the FIQ and VAS scores were assessed using the Cronbach's alpha. Data were analyzed using the SPSS software version 16.0 (SPSS Inc., Chicago, IL, USA).

Datawere analyzed using the GraphPad Prismversion 6.01 software (GraphPad Software Inc., CA, USA). Descriptive data were expressed in mean $\pm S D$. Initially, all measurements were evaluated using the D'AgostinoPearson Test for normality. Then, normally distributed data were analyzed. Comparisons of the mean values

(b)

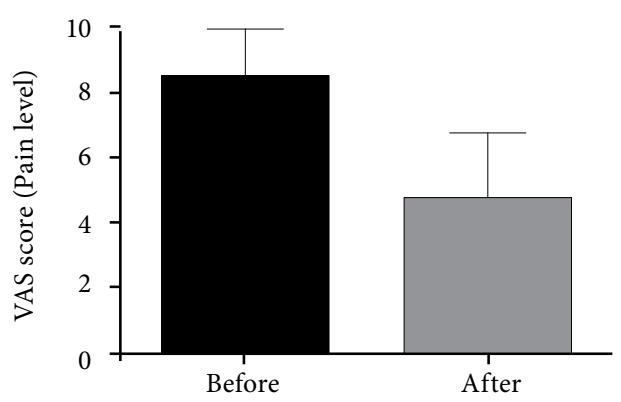

Figure 2. The (a) FIQ and (b) VAS-pain scores. Data are presented in mean (boxes) \pm SD (bars) based on the analysis of the D'Agostino and Pearson normality test. There was a significant decrease in these parameters following exercise (paired t-test: $\mathrm{p}<0.0001$ ). FIQ: Fibromyalgia Impact Questionnaire; VAS: Visual Analog Scale. 
of the FIQ and VAS scores were made using the paired t-test. All comparisons of PSD data of different control mechanisms in FMS patients were made with repeated measures analysis of variance (ANOVA), followed by the Tukey's corrected multiple comparison test. Multiplicity-adjusted p values were reported. Comparisons between the FMS patients and controls were made with one-way ANOVA of unpaired samples,

(a)

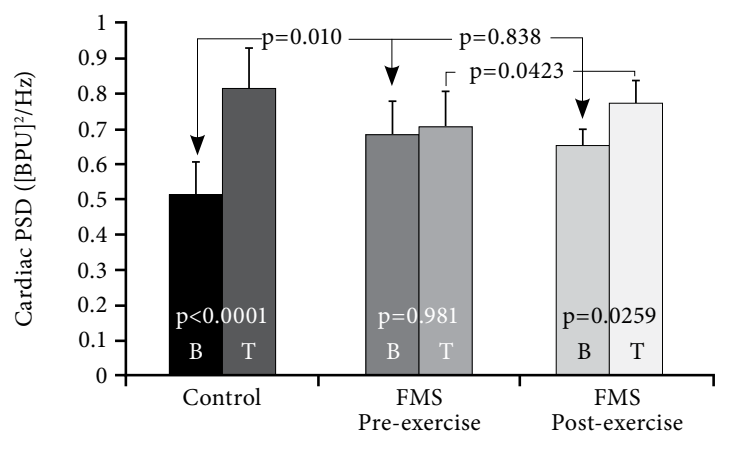

(b)

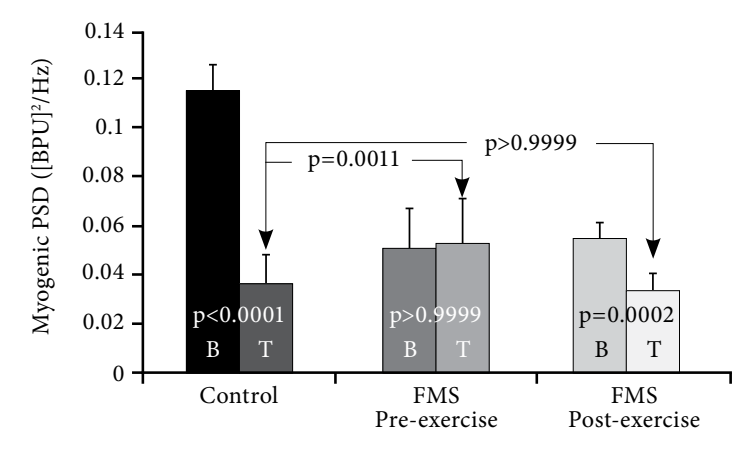

(c)

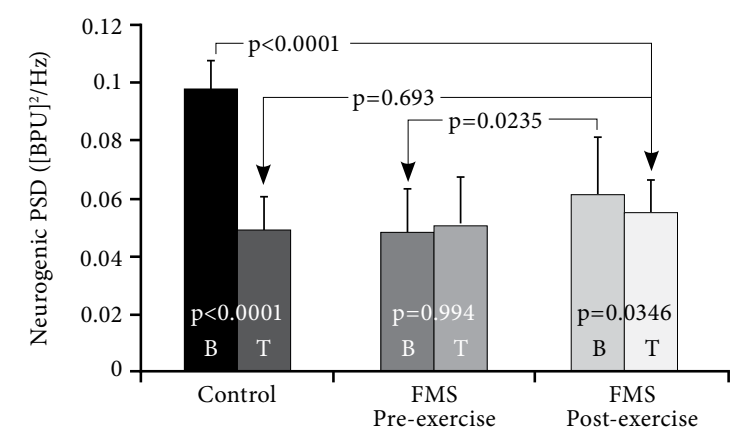

followed by the Tukey's corrected multiple comparison test. A $p$ value of $<0.05$ was considered statistically significant.

Linear regression analysis was performed to determine the effect of FMS severity and pain level on blood flow control mechanisms. Therefore, the PSD values of each control mechanism measured in the (d)

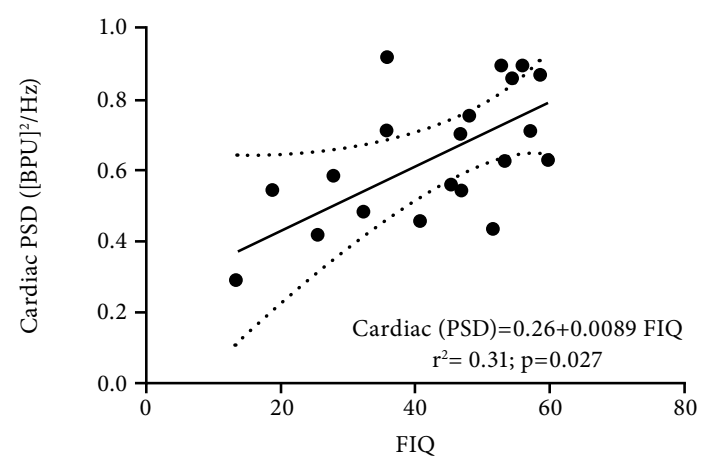

(e)

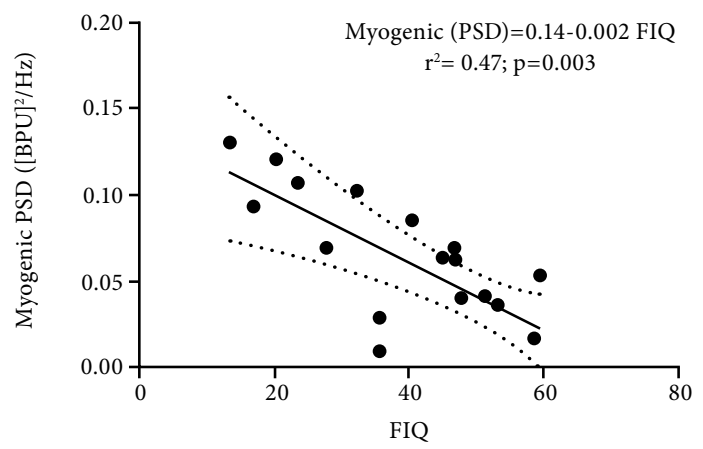

(f)

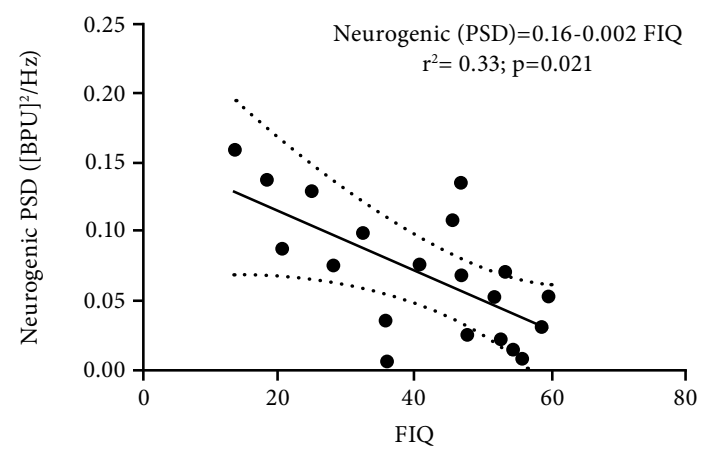

Figure 3. Relative power spectral density values of LDF signals, (a) indicating the influence of heart beat and (b) the activity of local control mechanisms; myogenic and (c) neurogenic. All values are presented in mean (boxes) \pm SD (bars). A comparison was made using the ANOVA and Tukey's multiple comparisons tests $(\mathrm{p}<0.0001)$. The straight line graphs indicate correlations between fibromyalgia impact questionnaire scores of patients and power spectral density values of control mechanisms: (d) cardiac, (e) myogenic and (f) neurogenic. The fitted regression line and upper and lower 95\% confidence limits are shown by the full and dotted lines respectively. ANOVA: Analysis of variance; B: Baseline; T: Response to local heat; BPU: Blood perfusion unit; LDF: Laser Doppler flowmetry, PSD: Power spectral density; FMS: Fibromyalgia syndrome; FIQ: Fibromyalgia Impact Questionnaire. 
(a)

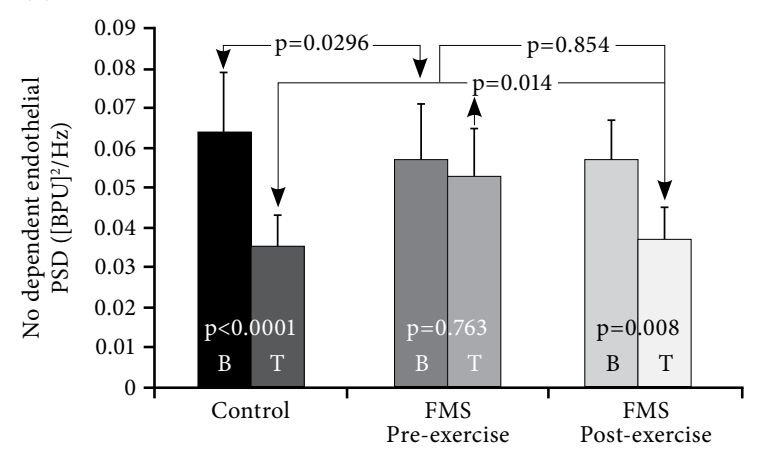

(b)

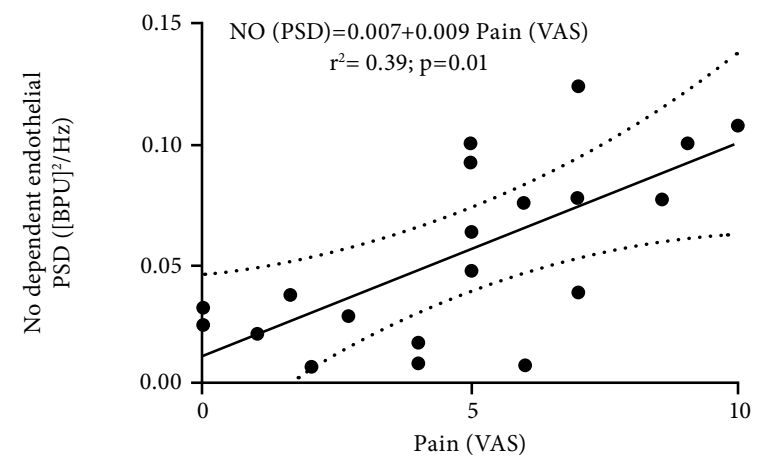

Figure 4. (a) Relative power spectral density values of LDF signals indicating the contribution of nitric oxide-dependent endothelial activity to microvascular control in baseline (b) condition and during response to local heat (T). All values are presented in mean (boxes) \pm SD (bars). A comparison was made using the ANOVA and Tukey's multiple comparisons tests $(\mathrm{p}<0.0001)$. (b) The straight line graph indicates correlation between pain scores of patients and power spectral density values of nitric oxide-dependent control mechanism. The fitted regression line and upper and lower 95\% CI are shown by the full and dotted lines respectively (b). ANOVA: Analysis of variance; LDF: Laser Doppler flowmetry; PSD: Power spectral density; NO: Nitric oxide; BPU: Blood perfusion unit; B: Baseline; T: Response to local heat; FMS: Fibromyalgia syndrome; VAS: Visual Analog Scale.

FMS patients were plotted against their FIQ scores or VAS levels. Therefore, each of the five different control mechanisms was plotted as a function of FIQ or VAS in a separate graph. The fitted regression lines to these experimental points and their upper and lower 95\% confidence limits were calculated using the multiple linear regression analysis.

\section{RESULTS}

There were no significant differences between the FMS and control groups in terms of age and the other parameters which are known to affect microvascular blood flow, including body mass index, blood pressure, and HR ( $p>0.05)$ (Table 1). However, we found a significant improvement in the clinical parameters (FIQ and VAS) of the FMS patients with exercise (Figure 2).

Baseline PSD values of cardiac control were significantly higher in the FMS group than the controls $(\mathrm{p}<0.001)$ (Figure 3a). Thermal $(\mathrm{T})$ hyperemia $(\mathrm{T})$-induced vasodilatation strengthened cardiac control mechanism in the control group, but not in the FMS group. Although it was not the same as in control, a significant response to local heating was observed in the FMS group after exercise training (Figure 3a). In addition, baseline values of post-exercise cardiac PSDs showed a significant positive correlation with postexercise FIQ scores (Figure 3d). On the other hand, there was no any other correlation between cardiac PSD values and FIQ scores.
Activity of myogenic (Figure $3 b$ ) and neurogenic (Figure 3c) control mechanisms were significantly different between the FMS and control groups $(\mathrm{p}<0.001)$. Myogenic and neurogenic responses to local heating $(\mathrm{T})$, which were seen in the control group, were unable to be observed in the FMS patients. However, these responses became significant in the FMS group after exercise training $(\mathrm{p}<0.05)$. In addition, there were negative correlations between the FIQ scores and two control mechanisms: myogenic (Figure 3e) and neurogenic (Figure 3f) following exercise.

Similarly, NO-dependent endothelial response to local heat, which was not the case in the FMS patients, became significant after exercise training in the control group (Figure 4). Different from the other control mechanisms, NO-dependent endothelial activity had a negative correlation with VAS scores in the FMS patients (Figure 4).

\section{DISCUSSION}

Cutaneous blood flow is controlled by oscillatory mechanisms of both local (myogenic, neurogenic and endothelial) and central (cardiac and respiratory) origins. It is conceivable that sedentary lifestyle and chronic widespread pain in FMS patients may adversely affect these control systems, thereby, contributing to an increased predisposition of microvascular impairment. However, these mechanisms have not been studied using the frequency domain methods in FMS patients, yet. Spectral analysis based on the FFT 
of LDF signals was used to evaluate the current state of these mechanisms.

On the other hand, exercise is a common recommendation in the management of FMS. Therefore, we conducted the present study to examine the impact of exercise training on microvascular functions. Before exercise, consistent with our hypothesis, there were weak responses to local heat (a vasodilator stimulus) in the FMS patients, compared to the healthy controls, and local control mechanisms were impaired in these patients. Following fourweek moderate aerobic exercise training, we found significant improvements in local mechanisms, and in the FIQ and VAS scores. In parallel to these improvements, baseline PSD values of myogenic and neurogenic control systems were shown to be negatively correlated, whereas baseline PSD values of cardiac influences were shown to be positively correlated with the FIQ scores. However, baseline PSD values of NO-dependent endothelial control system were positively correlated only with VAS scores. These results suggest an impaired microvascular function in FMS patients, depending on the severity of disease, as assessed by the FIQ and VAS scores. The apparent weakness in the microvascular response to local heating could be attributed to the existence of excessive baseline activities in myogenic, neurogenic, and NO-dependent endothelial control systems in patients with FMS. Thus, chronic exposure to pain and sedentary lifestyle may decrease the sensitivity of local mechanisms to the vasodilator stimuli. In contrast, exercise may protect microvascular beds against harmful effects of these conditions.

Periodic oscillations of the cutaneous perfusion with frequencies around $1 \mathrm{~Hz}$ originate from the pulsatile changes in the blood pressure due to periodic contraction of the heart. ${ }^{[23]}$ Vascular smooth muscle cells in the small arteries and arterioles respond to these acute changes in blood pressure via a mechanism known as the myogenic effect. ${ }^{[34]}$ Elevated pressure triggers contraction and narrows vascular beds to keep blood flow constant in capillaries. As a result, arterioles reduce the periodic variation in pressure and blood flow. Since the pulsatility is the leading risk factor for cardiovascular system, ${ }^{[35]}$ baseline cardiac signal and its PSD value should be as low as possible in healthy microvascular beds during the supine rest. However, baseline values of cardiac PSD were greater in the FMS patients in the present study $(p<0.0001)$. Furthermore, there was no significant change in cardiac PSD values in response to local heat, suggesting an impaired myogenic response, before exercise in the FMS group. Following exercise, cardiac PSD decreased, depending on the improvement in the FIQ scores, and a significant response was seen in the FMS patients.

Interestingly, baseline PSD values of myogenic and neurogenic control systems in the FMS patients were approximately similar to the response to local heat $(\mathrm{T})$ in the control group. Furthermore, these two control systems did not respond to local heat in the FMS patients. Due to the chronic exposure of FMS patients to a variety of stimuli, neural or not, our results suggest an impaired myogenic and neurogenic control in these patients, which could be explained by the reduced efficacy of these systems in these patients. Negative correlations between the FIQ scores and two local mechanisms in the present study also confirm aforementioned suggestion. Therefore, with improvement in the FIQ scores, baseline activities of myogenic and neurogenic mechanisms and baseline PSD values corresponding to these activities should be returned to normal, depending on the decrease in severity of symptoms through exercise. Although their baseline PSD values did not attain to the level of control group, responses of myogenic and neurogenic mechanisms to local heat were became statistically significant in the FMS patients following exercise. These results are also consistent with the findings of Kvernmo et al. ${ }^{[36]}$

Furthermore, several studies have shown that NOS activity ${ }^{[12]}$ and NO level are already high in FMS patients. ${ }^{[11,13,14]}$ Therefore, in case of increased NO, one would expect that the susceptibility/ sensitivity of a vascular system to a change in NO would be less. Although the local temperaturedependent mechanisms involve both axon reflex and local generation of $\mathrm{NO},{ }^{[27]}$ we found no change in the NO-dependent endothelial PSD values in response to local heat. This reduced sensitivity in the endothelial control system, which reveals an impaired NO-dependent endothelial activity, is also consistent with the finding of an excessive NO production in FMS patients..$^{[11,13,14]}$

In a healthy individual, one of the main mechanisms by which exercise training can positively affect the microvascular function is to enhance NO bioavailability. ${ }^{[37]}$ It has been shown that acute exercise significantly increases NO production and endothelial NO synthase expression. ${ }^{[38]}$ In contrary to the acute increase in NO production, if regular physical activity induces beneficial changes in the microvascular 
function, excessive NO should be reduced to normal levels following a sustained exercise period in FMS patients. Due to the presence of a positive correlation between the pain scores and endothelial PSD values, one may expect that the endothelial system should recover from their excessive baseline activity, depending on the reduction in the pain severity with exercise. Consistent with this finding, $\mathrm{NO}$-dependent endothelial response to local heat significantly increased following exercise in our study. An important conclusion to be drawn from this finding is, thus, that exercise have both ways to modulate/regulate $\mathrm{NO}$ production, and our results indicate that it reduces excessive NO production in FMS patients. Although this finding is consistent with the finding of Sarıfakığlu et al., ${ }^{[13]}$ further studies are needed to test this hypothesis.

Nonetheless, this study has some limitations. First, we were unable to measure NO levels in FMS patients. However, we found that the changes in the NO-dependent endothelial PSD values were NO-mediated. Second, although exercise program appeared to be successful to improve microvascular functions and symptom scores in FMS patients, a four-week period may not be sufficient to fully represent the necessary improvements. Third, an exercise program was unable to be applied to the control group; therefore, we were unable to make a comparison between the groups.

In conclusion, compared to healthy age-matched controls, FMS patients had impaired microvascular functions. These results indicate that moderateintensity aerobic exercise exerts a beneficial effect on the local control mechanisms, neurogenic, myogenic and endothelial, of microvascular beds and improves disease severity, as evidenced by the FIQ and VAS scores in FMS patients. Overall, our results highlight the importance of physical exercise as an adjuvant therapy in cardiovascular risk factor modification in FMS patients.

\section{Declaration of conflicting interests}

The authors declared no conflicts of interest with respect to the authorship and/or publication of this article.

\section{Funding}

The authors received no financial support for the research and/or authorship of this article.

\section{REFERENCES}

1. Clauw DJ, Arnold LM, McCarberg BH. The science of fibromyalgia. Mayo Clin Proc 2011;86:907-11.

2. Buskila D. Muscle pain in rehabilitation. Am J Phys Med Rehabil 2012;91:1101-3.
3. Friedberg F, Williams DA, Collinge W. Lifestyle-oriented non-pharmacological treatments for fibromyalgia: a clinical overview and applications with home-based technologies. J Pain Res 2012;5:425-35.

4. McLoughlin MJ, Colbert LH, Stegner AJ, Cook DB. Are women with fibromyalgia less physically active than healthy women? Med Sci Sports Exerc 2011;43:905-12.

5. Manson JE, Greenland P, LaCroix AZ, Stefanick ML, Mouton CP, Oberman A, et al. Walking compared with vigorous exercise for the prevention of cardiovascular events in women. N Engl J Med 2002;347:716-25.

6. Laufs U, Wassmann S, Czech T, Münzel T, Eisenhauer M, Böhm M, et al. Physical inactivity increases oxidative stress, endothelial dysfunction, and atherosclerosis. Arterioscler Thromb Vasc Biol 2005;25:809-14.

7. Jasperse JL, Laughlin MH. Endothelial function and exercise training: evidence from studies using animal models. Med Sci Sports Exerc 2006;38:445-54.

8. Higashi Y, Yoshizumi M. Exercise and endothelial function: role of endothelium-derived nitric oxide and oxidative stress in healthy subjects and hypertensive patients. Pharmacol Ther 2004;102:87-96.

9. Carville SF, Arendt-Nielsen L, Bliddal H, Blotman F, Branco JC, Buskila D, et al. EULAR evidence-based recommendations for the management of fibromyalgia syndrome. Ann Rheum Dis 2008;67:536-41.

10. Winkelmann A, Häuser W, Friedel E, Moog-Egan M, Seeger $D$, Settan M, et al. Physiotherapy and physical therapies for fibromyalgia syndrome. Systematic review, meta-analysis and guideline. Schmerz 2012;26:276-86. [Abstract]

11. Anbar M, Gratt BM. Role of nitric oxide in the physiopathology of pain. J Pain Symptom Manage 1997;14:225-54.

12. Cimen OB, Cimen MY, Yapici Y, Camdeviren H. Arginase, NOS activities, and clinical features in fibromyalgia patients. Pain Med 2009;10:813-8.

13. Sarıfakıoğlu B, Güzelant AY, Güzel EC, Güzel S, Kızıler AR. Effects of 12-week combined exercise therapy on oxidative stress in female fibromyalgia patients. Rheumatol Int 2014;34:1361-7.

14. Larson AA, Giovengo SL, Russell IJ, Michalek JE. Changes in the concentrations of amino acids in the cerebrospinal fluid that correlate with pain in patients with fibromyalgia: implications for nitric oxide pathways. Pain 2000;87:201-11.

15. Holowatz LA, Thompson-Torgerson CS, Kenney WL. The human cutaneous circulation as a model of generalized microvascular function. J Appl Physiol (1985) 2008;105:370-2.

16. Ozbebit FY, Esen F, Güleç S, Esen H. Evaluation of forearm microvascular blood flow regulation by laser Doppler flowmetry, iontophoresis, and curve analysis: contribution of axon reflex. Microvasc Res 2004;67:207-14.

17. Esen F, Aydin GS, Esen H. Detrended fluctuation analysis of laser Doppler flowmetry time series. Microvasc Res 2009;78:314-8.

18. Jeschonneck M, Grohmann G, Hein G, Sprott H. Abnormal microcirculation and temperature in skin above tender points in patients with fibromyalgia. Rheumatology (Oxford) 2000;39:917-21. 
19. MorfS, Amann-Vesti B, Forster A, FranzeckUK, Koppensteiner $\mathrm{R}$, Uebelhart $\mathrm{D}$, et al. Microcirculation abnormalities in patients with fibromyalgia - measured by capillary microscopy and laser fluxmetry. Arthritis Res Ther 2005;7:209-16.

20. Frödin T, Bengtsson A, Skogh M. Nail fold capillaroscopy findings in patients with primary fibromyalgia. Clin Rheumatol 1988;7:384-8.

21. Al-Allaf AW, Khan F, Moreland J, Belch JJ, Pullar T. Investigation of cutaneous microvascular activity and flare response in patients with fibromyalgia syndrome. Rheumatology (Oxford) 2001;40:1097-101.

22. Zhang Y, Ge HY, Yue SW, Kimura Y, Arendt-Nielsen L. Attenuated skin blood flow response to nociceptive stimulation of latent myofascial trigger points. Arch Phys Med Rehabil 2009;90:325-32.

23. Bernjak A, Clarkson PB, McClintock PV, Stefanovska A. Low-frequency blood flow oscillations in congestive heart failure and after betal-blockade treatment. Microvasc Res 2008;76:224-32.

24. Kvandal P, Landsverk SA, Bernjak A, Stefanovska A, Kvernmo HD, Kirkebøen KA. Low-frequency oscillations of the laser Doppler perfusion signal in human skin. Microvasc Res 2006;72:120-7.

25. Kvandal P, Landsverk SA, Bernjak A, Stefanovska A, Kvernmo HD, Kirkebøen KA. Low-frequency oscillations of the laser Doppler perfusion signal in human skin. Microvasc Res 2006;72:120-7.

26. Bennett RM, Friend R, Jones KD, Ward R, Han BK, Ross RL. The Revised Fibromyalgia Impact Questionnaire (FIQR): validation and psychometric properties. Arthritis Res Ther 2009;11:120.

27. Roustit M, Cracowski JL. Non-invasive assessment of skin microvascular function in humans: an insight into methods. Microcirculation 2012;19:47-64.

28. Bergholm R, Mäkimattila S, Valkonen M, Liu ML, Lahdenperä $\mathrm{S}$, Taskinen $\mathrm{MR}$, et al. Intense physical training decreases circulating antioxidants and endothelium- dependent vasodilatation in vivo. Atherosclerosis 1999;145:341-9.

29. Heylen E, Guerrero F, Mansourati J, Theron M, Thioub S, Saïag B. Effect of training frequency on endotheliumdependent vasorelaxation in rats. Eur J Cardiovasc Prev Rehabil 2008;15:52-8.

30. Goto C, Higashi Y, Kimura M, Noma K, Hara K, Nakagawa $\mathrm{K}$, et al. Effect of different intensities of exercise on endothelium-dependent vasodilation in humans: role of endothelium-dependent nitric oxide and oxidative stress. Circulation 2003;108:530-5.

31. Haram PM, Adams V, Kemi OJ, Brubakk AO, Hambrecht $\mathrm{R}$, Ellingsen $\mathrm{O}$, et al. Time-course of endothelial adaptation following acute and regular exercise. Eur J Cardiovasc Prev Rehabil 2006;13:585-91.

32. Haskell WL, Lee IM, Pate RR, Powell KE, Blair SN, Franklin $\mathrm{BA}$, et al. Physical activity and public health: updated recommendation for adults from the American College of Sports Medicine and the American Heart Association. Med Sci Sports Exerc 2007;39:1423-34.

33. Thomas EN, Blotman F. Aerobic exercise in fibromyalgia: a practical review. Rheumatol Int 2010;30:1143-50.

34. Davis MJ. Perspective: physiological role(s) of the vascular myogenic response. Microcirculation 2012;19:99-114.

35. O'Rourke MF, Hashimoto J. Mechanical factors in arterial aging: a clinical perspective. J Am Coll Cardiol 2007;50:1-13.

36. Kvernmo HD, Stefanovska A, Bracic M, Kirkebøen KA, Kvernebo K. Spectral analysis of the laser Doppler perfusion signal in human skin before and after exercise. Microvasc Res 1998;56:173-82.

37. Green DJ, Maiorana A, O'Driscoll G, Taylor R. Effect of exercise training on endothelium-derived nitric oxide function in humans. J Physiol 2004;561:1-25.

38. Whyte JJ, Laughlin MH. The effects of acute and chronic exercise on the vasculature. Acta Physiol (Oxf) 2010;199:441-50. 